\title{
Impacto psicológico da COVID-19 nos profissionais de saúde: revisão sistemática de prevalência
}

Psychological impact of COVID-19 on healthcare workers: prevalence systematic review Impacto psicológico de la COVID-19 en los profesionales de la salud: revisión sistemática de prevalencia

Liliana Sousa ${ }^{1}$ iD https://orcid.org/0000-0001-6445-0236

Jorge Miguel Albuquerque ${ }^{2}$ id https://orcid.org/0000-0002-3775-9980

Madalena Cunha ${ }^{3,4}$ iD https://orcid.org/0000-0003-0710-9220

Eduardo José Ferreira dos Santos ${ }^{1}$ id https://orcid.org/0000-0003-0557-2377

Como citar:

Sousa L, Albuquerque JM, Cunha M, Santos EJ. Impacto psicológico da COVID-19 nos profissionais de saúde: revisão sistemática de prevalência. Acta Paul Enferm. 2021;34:AAPE003775.

DOI

http://dx.doi.org/10.37689/actaape/2021AR03775

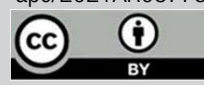

Descritores

Infeções por coronavirus; COVID-19; Saúde mental; Depressão; Ansiedade; Angústia psicológica;

Pessoal de saúde

Keywords

Coronavirus infections; COVID-19; Mental health; Depression; Anxiety; Psychological distress; Health

personnel

Descriptores

Infecciones por coronavirus; COVID-19; Salud mental; Depresión; Ansiedad; Distrés psicológico;

Personal de salud

Submetido 10 de Dezembro de 2020

Aceito

31 de Maio de 2021

Autor correspondente Eduardo José Ferreira dos Santos Email: ejf.santos87@gmail.com

\section{Resumo}

Objetivo: Sintetizar a prevalência de resultados psicológicos e de saúde mental dos profissionais de saúde que tratam doentes com COVID-19.

Métodos: Revisão sistemática da literatura. A busca dos estudos foi realizada nas plataformas PubMed, CINAHL e Scopus. Foram incluídos estudos em língua inglesa, portuguesa e espanhola, com data de publicação de 1 de dezembro de 2019 até 31 de julho de 2020. A revisão sistemática foi realizada com metaanálises binárias de efeito fixo de prevalência utilizando-se 0 STATA $® 15.0$ e método do inverso da variância com transformação Freeman-Tukey double arcsine.

Resultados: A estratégia de pesquisa identificou 38.657 registos, dos quais apenas cinco estudos foram selecionados e integraram o corpus final da revisão. A meta-análise de prevalências para a depressão foi de 27,5\% (IC95\%=25,9-29,3; $p<0,001$ ), ansiedade 26,8\% (IC95\%=25,1-28,5; $p<0,001$ ), insônia 35,8\% (IC95\%=33,8-37,9; $p=0,03$ ) e estresse $51,9 \%$ (IC95\%=49,6-54,3; $p<0,001)$. Em três dos estudos incluídos os profissionais de saúde relataram níveis de trauma vicário adicionalmente importante, estresse póstraumático, somatização e sintomas obsessivo-compulsivos.

Conclusão: A pandemia da COVID-19 apresenta um impacto psicológico muito significativo nos profissionais de saúde, assumindo importantes prevalências de depressão, ansiedade, insônia e estresse. Os profissionais de saúde da "linha de frente" são uma população particularmente vulnerável e merecem especial atenção/ intervenção.

\section{Abstract}

Objective: To synthesize the prevalence of psychological and mental health outcomes among healthcare professionals who are responsible for treating patients with COVID-19.

Methods: Systematic literature review. The literature search was carried out in the PubMed, CINAHL and Scopus databases. Studies written in English, Portuguese and Spanish and that were published between December $1^{\text {st }} 2019$ and July $31^{\text {st }} 2020$ were included. The systematic review was performed using fixed-effect meta-analysis of binary data with STATA®15.0 and inverse-variance method using Freeman-Tukey double arcsine transformation.

Results: The search strategy identified 38,657 records. Only five of those studies were selected and were included in the final review corpus. The meta-analysis conducted showed that the prevalence of depression reached $27.5 \%(95 \% \mathrm{Cl}=25.9-29.3 ; \mathrm{p}<0.001)$, the prevalence of anxiety was $26.8 \%(95 \% \mathrm{Cl}=25.1-28.5$; $\mathrm{p}<0.001)$, that of insomnia $35.8 \%(95 \% \mathrm{Cl}=33.8-37.9 ; \mathrm{p}=0.03)$ and the prevalence of stress amounted to

Centro Hospitalar e Universitário de Coimbra, Coimbra, Portugal.

¿Escola Superior de Saúde de Viseu, Viseu, Portugal.

Instituto Politécnico de Viseu, Escola Superior de Saúde, Viseu, Portugal.

"Universidade do Minho, Viseu, Portugal.

Conflitos de interesse: nada a declarar. 
$51.9 \%(95 \% \mathrm{Cl}=49.6-54.3 ; \mathrm{p}<0.001)$. Three of the studies included in the review show that healthcare professionals have also reported significant levels of vicarious traumatization, posttraumatic stress, somatization, and obsessive-compulsive symptoms.

Conclusion: The COVID-19 pandemic is found to have a very significant psychological impact on healthcare workers and is quite likely to lead to an important prevalence of depression, anxiety, insomnia, and stress. Frontline healthcare professionals are a particularly vulnerable group and deserve special attention/ intervention.

\section{Resumen}

Objetivo: Sintetizar la prevalencia de resultados psicológicos y de salud mental de los profesionales de la salud que tratan enfermos de COVID-19.

Métodos: Revisión sistemática de la literatura. La búsqueda de los estudios fue realizada en las plataformas PubMed, CINAHL y Scopus. Se incluyeron estudios en idioma inglés, portugués y español, con fecha de publicación entre 1 de diciembre de 2019 y 31 de julio de 2020. La revisión sistemática fue realizada con metaanálisis binarios de efecto fijo de prevalencia mediante la utilización del STATA®15.0 y el método del inverso de la varianza con transformación Freeman-Tukey double arcsine.

Resultados: A través de la estrategia de investigación se identificaron 38.657 registros, de los cuales solo cinco estudios fueron seleccionados e integraron el corpus final de la revisión. El metaanálisis de prevalencias de la depresión fue del 27,5 \% (IC95 \%=25,9-29,3; $p<0,001$ ), ansiedad 26,8 \% (IC95 \%=25,128,$5 ; p<0,001$ ), insomnio 35,8 \% (IC95 \%=33,8-37,9; $p=0,03$ ) y estrés 51,9\% (IC9 $5 \%=49,6-54,3 ; p<0,001$ ). En tres de los estudios incluidos, los profesionales de la salud relataron niveles importantes de trauma vicario, estrés postraumático, somatización y síntomas obsesivo-compulsivos.

Conclusión: La pandemia de COVID-19 presenta un impacto psicológico muy significativo en los profesionales de la salud, con importantes prevalencias de depresión, ansiedad, insomnio y estrés. Los profesionales de la salud de la "línea de frente" son una población particularmente vulnerable y merecen especial atención/intervención.

\section{Introdução}

Desde dezembro de 2019, o surto da doença de Coronavírus de 2019 (COVID-19) que surgiu na cidade de Wuhan na China levou os sistemas de saúde à beira da falência em todo o mundo ${ }^{(1-3)}$ e colocou os profissionais de saúde sob uma carga de trabalho e estresse avassaladores. ${ }^{(4-6)}$

A 30 de janeiro de 2020, a Organização Mundial de Saúde declarou estado de emergência de saúde pública de preocupação internacional. Mais tarde, em 11 de março de 2020, o surto foi caracterizado como uma pandemia, principalmente porque o vírus era altamente infecioso e com índices de propagação exponenciais em todo o mundo. ${ }^{(7)}$

A COVID-19 aparentava ser menos virulenta quando comparada com outros vírus, contudo, os seus longos períodos de incubação traduzem-se num número significativo de portadores assintomáticos e pré-sintomáticos. ${ }^{(8-10)} \mathrm{O}$ número de casos confirmados cresce continuamente a nível mundial, e oito meses após o surto, foram notificados mais de 126 milhóes de casos e mais de 2,7 milhóes de mortes. $^{(11)}$

Os profissionais da saúde têm trabalhado eficazmente contra a COVID-19, enfrentando um risco substancial de infeção (designada de SARS-CoV-2), com enormes sacrifícios pessoais e profissionais. $(4,5,12,13)$ Devido às transmissóes assintomáticas que aumentam o risco de infeção hospitalar e à exposição, os profissionais de saúde são uma população particularmente de risco. ${ }^{(14)}$

Por outro lado, estes profissionais diretamente envolvidos no diagnóstico, tratamento e cuidados de doentes com COVID-19, (comumente designados de profissionais da "linha da frente"), são colocados sob exigências extremas que ameaçam a sua resiliência profissional.

O estresse e o conflito emocional nestas circunstâncias podem surgir de sobrecarga de trabalho, falta de proteçáo pessoal e outros equipamentos, separação prolongada da família, aumento implacável do número de casos e mortes, pressão das notícias e das redes sociais e da exposição ao risco de infeção, entre muitos outros. ${ }^{(4,5,12,13)}$ Isto repercute-se em diversos resultados/ outcomes psicológicos e de saúde mental.

Como a evidência nesta área se encontra dispersa, procurou-se sintetizar a prevalência de resultados psicológicos e de saúde mental dos profissionais de saúde que tratam doentes com COVID-19. Para isso, definiu-se como questão de investigação: Quais são os impactos psicológicos que manifestam os profissionais de saúde que estiveram diretamente envolvidos com doentes com COVID-19? Para esse efeito definimos "resultados psicológicos e de saúde mental" como qualquer perturbação psicológica adquirida e de que é caso a ansiedade, a depressão, o estresse, insônia, entre outros. ${ }^{(4,5,12,13)}$ 
Uma pesquisa preliminar realizada no dia 1 de julho de 2020 na JBI Database of Systematic Reviews and Implementation Reports, Cochrane Database of Systematic Reviews, PROSPERO e PubMed revelou que não havia nenhuma outra revisão sistemática publicada ou em curso.

\section{Métodos}

A revisão sistemática de prevalência e incidência com meta-análise seguiu o método da Joanna Briggs Institute $^{(15)}$ e foi redigida de acordo com o Preferred reporting items for systematic reviews and meta-analyses (PRISMA). ${ }^{(16)}$

O protocolo da revisão foi realizado e seguido pelos autores embora não tenha sido publicado e/ ou registado. Contudo, o mesmo pode ser enviado mediante pedido.

A pesquisa foi realizada no dia 31 de julho de 2020 nas plataformas PubMed, CINAHL e Scopus. Foram incluídos estudos em língua inglesa, portuguesa e espanhola, com data de publicação de 1 de dezembro de 2019 até 31 de julho de 2020. O determinante da escolha deste intervalo de tempo foi a data de início da pandemia COVID-19, pois só posteriormente começaram a emergir estudos sobre o tema em apreço.

Foram considerados os seguintes critérios de inclusão:

- PARTICIPANTES: Foram considerados todos os estudos que se refiram a profissionais de saúde que contactaram/trataram de doentes com COVID-19;

- CONDIÇÃO: Perturbaçóes psicológicas e/ou de saúde mental adquiridas em tempo de pandemia COVID-19: especificamente a ansiedade, a depressão, o estresse e a insônia;

- CONTEXTO: Sem restriçóes podendo tratar-se de ambiente hospitalar, ambulatório, entre outros.

- TIPO DE ESTUDOS: Estudos de natureza quantitativa, nomeadamente estudos quantitativos primários não randomizados, e estudos mistos, com possibilidade de extrair os dados quantitativos de forma isolada.
A estratégia de pesquisa aplicada à Pubmed foi: " $(((((C O V I D-19[$ Title/Abstract] $)$ OR (coronavirus*[Title/Abstract]) OR (SARS-CoV$2[$ Title/Abstract])) AND ((“depression”[MeSH Terms]) AND ("anxiety”[MeSH Terms]) OR (Insomnia[Title/Abstract]) OR (distress[Title/ Abstract]) OR (stress*[Title/Abstract]) OR ("mental health"[MeSH Terms])) AND ((healthprofessional*[Title/Abstract])))) OR (nurs*[Title/ Abstract])) OR (physician*[Title/Abstract])". Nas restantes bases de dados esta estratégia foi adaptada aos léxicos específicos.

Após a pesquisa, todas as citaçóes identificadas foram transferidas para o Endnote V7.7.1 (Clarivate Analytics, PA, EUA) e os duplicados removidos. A fim de avaliar a sua elegibilidade, os títulos e resumos foram analisados por dois revisores independentes (LS e JA). Na ausência de consenso foi incluído um terceiro revisor (ES) como critério de desempate. O Endnote V7.7.1 também foi utilizado como ferramenta de registro de cegamento dos revisores. Após a remoção dos duplicados, a biblioteca dos artigos foi distribuída pelos revisores para seleção independente e após esse procedimento as bibliotecas foram reconciliadas para análise da concordância da seleção.

A avaliação da qualidade dos estudos foi realizada por dois revisores independentes (LS e JA) através do instrumento "Checklist for Prevalence studies". ${ }^{(17)}$ Este instrumento inclui as seguintes questóes $(\mathrm{Q})$ : Q1. A amostra foi adequada para abordar a população alvo? Q2. Os participantes no estudo foram recrutados de uma forma adequada? Q3. O tamanho da amostra foi adequado? Q4. Os temas e contexto do estudo foram descritos em pormenor? Q5. A análise dos dados foi realizada com cobertura suficiente da amostra identificada? Q6. Foram utilizados métodos válidos para a identificação da condição? Q7. A condição foi medida de uma forma padrão e fiável para todos os participantes? Q8. Houve uma análise estatística adequada? Q9. A taxa de resposta foi adequada, e se não foi, a baixa taxa de resposta foi gerida apropriadamente? As respostas possíveis incluem sim, não ou não aplicável/ incerto. $\mathrm{O}$ cálculo das porcentagens é realizado para cada questão aplicando a fórmula: $\%$ Sim = número de sim respondidos / número de es- 
tudos. Valores de $100 \%$ indicam o cumprimento absoluto do critério de qualidade avaliado pela questão. Quanto mais elevada a porcentagem maior a qualidade. ${ }^{(17)} \mathrm{Na}$ ausência de consenso foi incluído um terceiro revisor (ES) como critério de desempate. Após a avaliação crítica, todos os estudos foram incluídos independentemente dos resultados. No entanto, os resultados da avaliaçáo crítica foram considerados na síntese das provas e relatados sob forma narrativa e tabelar.

Os dados foram extraídos por dois revisores independentes (LS e JA) e foi utilizado um instrumento de colheita de dados especialmente construído pelos autores para minimizar o risco de viés. A presença de desacordo entre os revisores foi resolvida com a inclusão de um terceiro revisor (ES). Os resultados foram agrupados numa tabela e objeto de síntese narrativa.

Por fim, foram realizadas meta-análises binárias de efeito fixo de prevalência através do programa STATA $^{\oplus} 15.0$ e método do inverso da variância com transformação Freeman-Tukey double arcsine. A heterogeneidade foi avaliada pelos testes do qui-quadrado e $I^{2}$. As análises estatísticas incluíram modelos de efeitos aleatórios apenas na presença de heterogeneidade moderada a elevada $\left(I^{2}>50 \%\right)$ e, na sua ausência, modelos de efeitos fixos. ${ }^{(18)}$

\section{Resultados}

A estratégia de pesquisa identificou um total de 38.657 registos dos quais 963 eram duplicados. Após leitura de todos os títulos e resumo foi possível excluir 37.602 por não se referirem especificamente ao tema em estudo. Os artigos remanescentes $(n=92)$ foram lidos integralmente e apreciados tendo por base os critérios de inclusáo definidos, que levou à exclusão de 87, devido, maioritariamente, às características dos participantes e condições definidas. Os 5 artigos apurados neste processo foram submetidos a avaliação crítica e constituíram o corpus final da revisão. Apenas 3 artigos foram incluídos na meta-análise devido à heterogeneidade estatística. Na figura 1 é apresentado o flowchart que resume o processo de seleção e inclusão dos estudos.

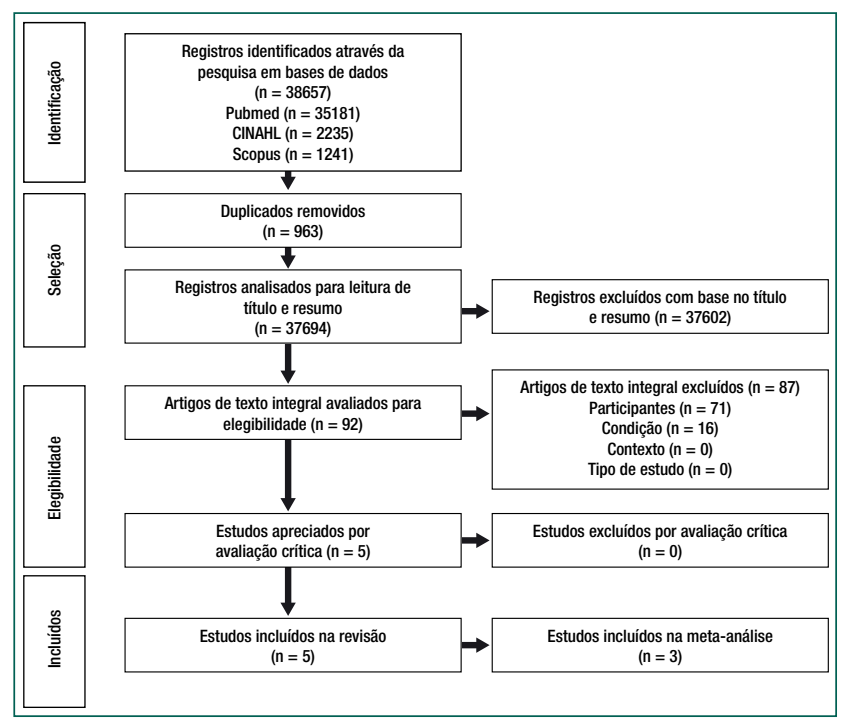

Figura 1. Flowchart da seleção e processo de inclusão dos estudos

Os resultados da avaliação crítica encontram-se sumariados no quadro 1 . Houve concordância entre os revisores para incluir os cinco estudos avaliados, pois foram considerados de elevada qualidade.

Quadro 1. Resultados da avaliação crítica dos estudos elegíveis

\begin{tabular}{|l|c|c|c|c|c|c|c|c|c|}
\hline Estudos & Q1 & Q2 & Q3 & Q4 & Q5 & Q6 & Q7 & Q8 & Q9 \\
\hline Lai et al., 2020(4) & S & S & S & S & S & S & S & S & S \\
\hline Li et al., 2020 (5) & S & S & S & S & S & S & S & S & S \\
\hline Xiao et al., 2020(6) & S & S & S & S & S & S & S & S & S \\
\hline Tan et al., 2020(12) & S & S & S & NA & S & S & S & S & S \\
\hline Zhang et al., 2020 (13) & S & S & S & S & S & S & S & S & S \\
\hline \% Sim & 100 & 100 & 100 & 80 & 100 & 100 & 100 & 100 & 100 \\
\hline
\end{tabular}

S - Sim; N - Não; NA - Não/Aplicável ou incerto

Todos os estudos incluídos são estudos observacionais e transversais cujas principais características foram sintetizadas no quadro 2 . Nenhum estudo teve fonte de financiamento.

\section{Meta-análise}

A meta-análise binária de efeito fixo de prevalência foi realizada em três estudos ${ }^{(4,12,13)}$ e contou com uma amostra de 2.654 profissionais de saúde. A prevalência combinada para a depressão foi de $27,5 \%$ (IC $95 \%=25,9-29,3 ; p<0,001$ ), para a ansiedade de $26,8 \%$ (IC 95\%=25,1-28,5; $p<0,001$ ), para a insônia de $35,8 \%$ (IC 95\%=33,8-37,9; $p=0,03$ ) e para o estresse de $51,9 \%$ (IC 95\%=49,6-54,3; $p<0,001$ ). Os resultados das meta-análises por outcome (depressão, ansiedade, insônia e estresse) são apresentados na figura 2 . 
Quadro 2. Características dos estudos incluídos na revisão

\begin{tabular}{|c|c|c|c|c|c|}
\hline Estudo/ País & Tipo de estudo & $\begin{array}{l}\text { População/ } \\
\text { Contexto }\end{array}$ & Condição & Resultados & Conclusões \\
\hline $\begin{array}{l}\text { Lai et al., } 2020^{(4)} \\
\text { (China) }\end{array}$ & $\begin{array}{l}\text { Estudo } \\
\text { transversal }\end{array}$ & $\begin{array}{l}1257 \text { profissionais } \\
\text { de saúde }(39,2 \% \\
\text { médicos e } 60,8 \% \\
\text { enfermeiros) / } \\
\text { hospitalar }\end{array}$ & $\begin{array}{l}\text { Depressão, } \\
\text { ansiedade, } \\
\text { insônia e } \\
\text { estresse }\end{array}$ & $\begin{array}{l}\text { - Os sintomas prevalentes foram: depressão }(50,4 \%) \text {, } \\
\text { ansiedade }(44,6 \%) \text {, insônia ( } 34,0 \%) \text {, e estresse }(71,5 \%) \text {. } \\
\text { - Os profissionais de saúde da "linha da frente" revelam } \\
\text { maior risco de sintomas de depressão (OR=1,52; } \\
\text { IC95\%=1,11-2,09; } p=0.01) \text {, ansiedade }(0 \mathrm{R}=1,57 \\
\text { IC } 95 \%=1,22-2,02 ; p<0.001) \text {, insônia }(0 \mathrm{R}=2,97 ; \\
\mathrm{IC} 95 \%=1,92-4,60 ; p<0.001) \text {, e estresse }(0 \mathrm{R}=1,60 ; \\
\mathrm{IC} 95 \%=1,25-2,04 ; p<0.001) \text {. }\end{array}$ & $\begin{array}{l}\text { - Os participantes referiram ter sofrido uma } \\
\text { carga psicológica, especialmente os enfermeiros, } \\
\text { mulheres, as de Wuhan, e profissionais de saúde } \\
\text { da "linha da frente" diretamente envolvidos no } \\
\text { diagnóstico, tratamento, e cuidados a doentes com } \\
\text { COVID-19. } \\
\text { - Devem ser implementadas intervenções } \\
\text { promotoras da saúde mental. }\end{array}$ \\
\hline $\begin{array}{l}\text { Li et al., 2020(5) } \\
\text { (China) }\end{array}$ & $\begin{array}{l}\text { Estudo } \\
\text { transversal }\end{array}$ & $\begin{array}{l}526 \text { enfermeiros e } \\
214 \text { população em } \\
\text { geral/ hospitalar }\end{array}$ & Trauma vicário & $\begin{array}{l}\text { - Os níveis de trauma vicário (respostas fisiológicas e } \\
\text { psicológicas), dos enfermeiros da "linha da frente", foram } \\
\text { significativamente inferiores às dos enfermeiros que não } \\
\text { pertencem à "linha da frente" ( } p<0,001) \text {. } \\
\text { - Os níveis de trauma vicário da população geral foram } \\
\text { significativamente superiores às dos enfermeiros da "linha da } \\
\text { frente" ( } p<0,001) \text {; não sendo observada diferença estatística } \\
\text { em comparação com os enfermeiros que não pertencem à } \\
\text { "linha da frente" ( } p>0,05) \text {. }\end{array}$ & $\begin{array}{l}\text { - Deve ser dada maior atenção aos problemas } \\
\text { psicológicos dos profissionais de saúde, } \\
\text { especialmente dos enfermeiros não pertencentes à } \\
\text { "linha da frente", e da população geral relacionados } \\
\text { com a pandemia COVID-19. } \\
\text { - São necessárias estratégias precoces de } \\
\text { prevenção e tratamento de trauma nos profissionais } \\
\text { de saúde e no público em geral. }\end{array}$ \\
\hline $\begin{array}{l}\text { Xiao et al., 2020 } \\
\text { (China) }\end{array}$ & $\begin{array}{l}\text { Estudo } \\
\text { transversal }\end{array}$ & $\begin{array}{l}180 \text { médicos/ } \\
\text { hospitalar }\end{array}$ & $\begin{array}{l}\text { Ansiedade, } \\
\text { insônia, estresse, } \\
\text { apoio social e } \\
\text { autoeficácia }\end{array}$ & $\begin{array}{l}\text { - } 0 \text { nível de apoio social dos médicos foi associado } \\
\text { significativamente à autoeficácia e à qualidade do sono e } \\
\text { negativamente associado aos níveis de ansiedade e estresse. } \\
\text { - Os níveis de ansiedade foram associados significativamente } \\
\text { aos níveis de estresse e tiveram um impacto negativo na } \\
\text { autoeficácia e na qualidade do sono. } \\
\text { - Ansiedade, estresse e autoeficácia foram variáveis } \\
\text { mediadoras do apoio social e da qualidade do sono. }\end{array}$ & $\begin{array}{l}\text { - Os médicos tiveram níveis de ansiedade, estresse } \\
\text { e autoeficácia que dependeram da qualidade do } \\
\text { sono e do apoio social. }\end{array}$ \\
\hline $\begin{array}{l}\text { Tan et al., 2020 } \\
\text { (Singapura) }\end{array}$ & \begin{tabular}{|l} 
Estudo \\
transversal
\end{tabular} & $\begin{array}{l}470 \text { profissionais de } \\
\text { saúde (296 médicos } \\
\text { e } 174 \text { não médicos) } \\
\text { / hospitalar }\end{array}$ & $\begin{array}{l}\text { Depressão, } \\
\text { ansiedade e } \\
\text { estresse }\end{array}$ & $\begin{array}{l}\text { - 14,5\% dos participantes manifestaram ansiedade, 8,9\% } \\
\text { depressão, } 6,6 \% \text { estresse, e 7,7\% estresse pós-traumático. } \\
\text { - A prevalência da ansiedade foi maior entre os profissionais } \\
\text { de saúde não médicos ( } 20,7 \% \text { vs } 10,8 \% \text {; rácio de } \\
\text { prevalência ajustado=1,85; IC95\%=1,15 a 2,99; } p=0,01) \text {. } \\
\text { - Foram observados scores médios mais elevados de } \\
\text { ansiedade e de impacto de eventos em profissionais de } \\
\text { saúde não médicos. }\end{array}$ & $\begin{array}{l}\text { - Os profissionais de saúde não médicos estiveram } \\
\text { em maior risco de distúrbios psicológicos durante } 0 \\
\text { surto da COVID-19. } \\
\text { - A aplicação de intervenções psicológicas precoces } \\
\text { dirigidas a este grupo vulnerável podem ser } \\
\text { benéficas. }\end{array}$ \\
\hline $\begin{array}{l}\text { Zhang et al., } \\
2020^{(13)} \\
\text { (China) }\end{array}$ & $\begin{array}{l}\text { Estudo } \\
\text { transversal }\end{array}$ & $\begin{array}{l}2182 \text { Profissionais } \\
\text { de saúde (927 } \\
\text { médicos e } 1255 \text { não } \\
\text { médicos) / hospitalar }\end{array}$ & $\begin{array}{l}\text { Insônia, } \\
\text { ansiedade, } \\
\text { depressão } \\
\text { e sintomas } \\
\text { somáticos e } \\
\text { obsessivo- } \\
\text { compulsivos }\end{array}$ & $\begin{array}{l}\text { - Em comparação com os profissionais de saúde não } \\
\text { médicos, os médicos tiveram uma prevalência mais elevada } \\
\text { de insônia ( } 38,4 \text { vs } 30,5 \%, p<0,01) \text {, ansiedade }(13,0 \\
\text { vs } 8,5 \%, p<0,01) \text {, depressão }(12,2 \text { vs } 9,5 \% ; p<0,04) \text {, } \\
\text { somatização }(1,6 \text { vs } 0,4 \% ; p<0,01) \text {, e sintomas obsessivo- } \\
\text { compulsivos }(5,3 \text { vs } 2,2 \% ; p<0,01) \text {. } \\
\text { - Viver em zonas rurais, ser do sexo feminino, e possuir risco } \\
\text { de contacto com doentes COVID-19 foram os fatores de risco } \\
\text { mais comuns para insônia, ansiedade, sintomas obsessivo- } \\
\text { compulsivos, e depressão ( } p<0,01 \text { ou } 0,05) \text {. }\end{array}$ & $\begin{array}{l}\text { - Durante o surto da COVID-19, os profissionais de } \\
\text { saúde tiveram problemas psicossociais e fatores de } \\
\text { risco para os desenvolver. }\end{array}$ \\
\hline
\end{tabular}

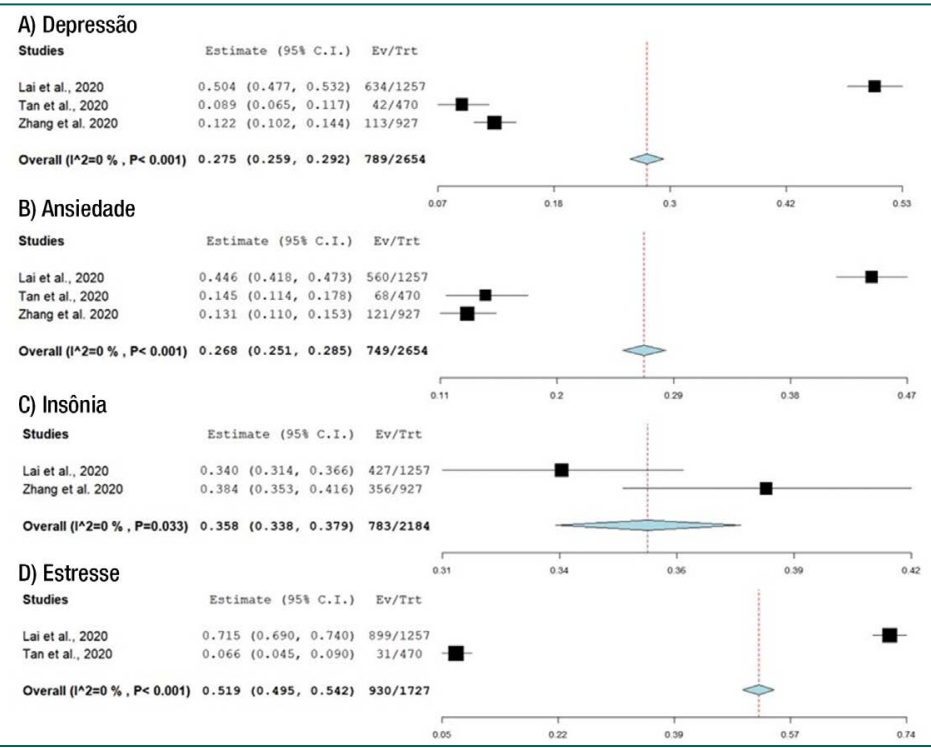

Figura 2. Meta-análises por outcome 
Dado que os estudos incluídos na meta-análise são de elevada qualidade não existe impacto na escolha dos modelos e/ ou pressupostos da meta-análise. Verificamos que não existe heterogeneidade entre os estudos incluídos $\left(I^{2}=0 \%\right)$ o que atesta a utilização dos modelos de efeitos fixos em todas as análises. A análise do viés de publicação não foi realizada pela inexistência de pelo menos 10 estudos o que nos permitiria realizar o funnel plot. ${ }^{(19)}$

\section{Discussão}

Apesar de atualmente já ser reconhecido o potencial impacto psicológico e de saúde mental nos profissionais de saúde que tratam doentes com COVID-19, ${ }^{(4,5,12,13)}$ os estudos não são concordantes. Isto motivou a realização desta revisão sistemática que, pela primeira vez, sintetizou a prevalência dos principais resultados (outcomes), aportando recomendaçôes relevantes para a prática clínica.

A meta-análise de prevalências para a depressão foi de $27,5 \%$, para a ansiedade de $26,8 \%$, para a insônia de $35,8 \%$ e de $51,9 \%$ para o estresse. Adicionalmente três dos estudos incluídos aportam que os profissionais de saúde relataram: níveis de trauma vicário importantes, ${ }^{(5)}$ sintomas de estresse pós-traumático, ${ }^{(12)}$ somatização e sintomas obsessivo-compulsivos. ${ }^{(13)}$

Estes resultados demostram claramente que os profissionais de saúde constituem um grupo particularmente suscetível para desenvolver problemas psicológicos. Mas ainda é controverso se esta realidade é mais prevalente em serviços ditos "da linha da frente" ou não, porque os estudos apontam conclusóes discrepantes. ${ }^{(4,5)}$ Por outro lado, ser enfermeiro, o genero feminino e a presença de comorbilidades, são aparentemente fatores de risco de psicopatologia ${ }^{(4,5,12)}$ mas isso também carece de confirmação futura.

Atendendo a prevalência dos problemas psicológicos citados, e a que atualmente a pandemia de COVID-19 se mantem sem tratamento que demonstre segurança e eficácia clínica duradoura, ${ }^{(20)} \mathrm{e}$ que os efeitos da vacinação generalizada ainda não são sentidos, é expectável que os problemas psico- lógicos dos profissionais de saúde se mantenham, ou venham mesmo a agravar, dada a exposição prolongada no tempo. Nesse sentido, é prioritário desenvolver intervençóes psicológicas precoces, que visem a promoção da saúde mental e resiliência e se necessário tratamento psicológico, dos profissionais de saúde como meio de os proteger. ${ }^{(4-6,12,13)}$

Os pontos fortes desta revisão sistemática incluíram os critérios rigorosos de inclusão, a seleção de estudos de qualidade e a rigorosa extração e síntese de dados. Contudo, esta revisão apresenta algumas limitaçóes como a inclusão de estudos referentes unicamente a um único país (China), a língua de publicação e a estratégia de pesquisa ser focada em apenas 3 bases de dados. Ainda assim, estas limitaçóes podem-se assumir como esperadas pois como a pandemia surgiu na China a maioria dos estudos têm essa proveniência. Em relação ao número de bases de dados utilizadas e que não incluiu, por exemplo, a literatura cinzenta é de referir que os estudos realizados sobre o tema COVID-19 foram disponibilizados em acesso livre e/ou repositórios dedicados na Pubmed como medida de acesso rápido à ciência. Por fim, apesar de existirem alguns estudos sobre o impacto psicológico da COVID-19 na população em geral, estes ainda não se encontram sintetizados nem apontam resultados claros. Esse fato inviabiliza estabelecer uma "verdadeira condição de risco" por ser profissional de saúde, pois as prevalências do impacto obtidas para estes profissionais não podem ser comparadas com a população em geral.

Como recomendações para a investigação futura sugere-se realizar uma revisão sistemática que sintetize a prevalência dos resultados psicológicos e de saúde mental na população em geral, para que as inferências de risco possam ser estabelecidas.

\section{Conclusão}

A pandemia da COVID-19 apresenta um impacto psicológico muito significativo nos profissionais de saúde, assumindo importantes prevalências de depressão, ansiedade, insônia e estresse. Os profissionais de saúde da "linha de frente" são uma popu- 
lação particularmente vulnerável e merecem especial atenção/intervenção. Como implicaçôes para a prática sugerimos a implementação de intervençóes psicológicas, que devem ser amplamente disponibilizadas e promovidas proativamente, como meio de proteger esta força de trabalho essencial e, com isso, garantir que eles possam continuar a satisfazer as extenuantes exigências que lhes são impostas em prol da saúde pública. Como implicaçóes para a investigação é recomendado que sejam realizados mais estudos sobre este tema e que posteriormente esta revisão sistemática seja atualizada.

\section{Agradecimentos}

Este trabalho foi financiado por Fundos Nacionais através da FCT - Fundação para a Ciência e a Tecnologia, I.P., no âmbito do projeto INVEST Refa: fab8899e-5c94-4c33-9807-f54cdc93b053 e projeto Refa UIDB/00742/2020. Os autores gostariam de reconhecer o suporte da Unidade de Investigação em Ciências da Saúde: Enfermagem (UICISA:E) e, adicionalmente ao Instituto Politécnico de Viseu.

\section{Referências}

1. Huang $C$, Wang $Y, L i X$, Ren $L, Z$ Zhao J, Hu Y, et al. Clinical features of patients infected with 2019 novel coronavirus in Wuhan, China. Lancet. 2020;395(10223):497-506.

2. Lu H, Stratton CW, Tang YW. Outbreak of pneumonia of unknown etiology in Wuhan, China: the mystery and the miracle. J Med Virol. 2020;92(4):401-2.

3. World Health Organization (WHO). WHO Director-general's remarks at the media briefing on 2019-nCoV on 11 February 2020. Geneva: WHO; 2021 [cited 2021 Mayo 16]. Available from: https://www.who.int/ $\mathrm{dg} /$ speeches/detail/who-director-general-s-remarks-at-the-mediabriefing-on-2019-ncov-on-11-february-2020

4. Lai J, Ma S, Wang Y, Cai Z, Hu J, Wei N, et al. Factors Associated With Mental Health Outcomes Among Health Care Workers Exposed to Coronavirus Disease 2019. JAMA Netw Open 2020;3(3):e203976-e.

5. Li Z, Ge J, Yang M, Feng J, Qiao M, Jiang R, et al. Vicarious traumatization in the general public, members, and non-members of medical teams aiding in COVID-19 control. Brain Behav Immun. 2020;88:916-919.
6. Xiao H, Zhang Y, Kong D, Li S, Yang N. The Effects of Social Support on Sleep Quality of Medical Staff Treating Patients with Coronavirus Disease 2019 (COVID-19) in January and February 2020 in China. Med Sci Monit. 2020;26:e923549.

7. World Health Organization (WHO). WHO Director-General's Remarks at the Media Briefing on COVID-19- 11 March 2020. Geneva: WHO; 2021 [cited 2021 Mayo 16]. Available from: https://www.who.int/dg/ speeches/detail/who-director-general-s-opening-remarks-at-themedia-briefing-on-covid-19---11-march-2020

8. Chen J. Pathogenicity and transmissibility of 2019-nCoV-A quick overview and comparison with other emerging viruses. Microbes Infect. 2020;22(2):69-71.

9. Mahase E. Coronavirus: covid-19 has killed more people than SARS and MERS combined, despite lower case fatality rate. BMJ. 2020;368:m641.

10. Zou L, Ruan F, Huang M, Liang L, Huang H, Hong Z, et al. SARS-CoV-2 Viral Load in Upper Respiratory Specimens of Infected Patients. N Engl J Med. 2020;382(12):1177-9.

11. World Health Organization (WHO). Weekly epidemiological update on COVID-19 - 30 March 2021 2020. Geneva:WHO; 2021. Available from: https://www.who.int/emergencies/diseases/novel-coronavirus-2019/ situation-reports/

12. Tan BY, Chew NW, Lee GK, Jing M, Goh Y, Yeo LL, et al. Psychological Impact of the COVID-19 Pandemic on Health Care Workers in Singapore. Ann Intern Med. 2020;173(4):317-20.

13. Zhang WR, Wang K, Yin L, Zhao WF, Xue Q, Peng M, et al. Mental Health and Psychosocial Problems of Medical Health Workers during the COVID-19 Epidemic in China. Psychother Psychosom. 2020;89(4):242-50.

14. Cho SY, Kang JM, Ha YE, Park GE, Lee JY, Ko JH, et al. MERS-CoV outbreak following a single patient exposure in an emergency room in South Korea: an epidemiological outbreak study. Lancet. 2016;388(10048):994-1001.

15. Munn Z, Moola S, Lisy K, Riitano D, Tufanaru C. Chapter 5: Systematic reviews of prevalence and incidence. In: Aromataris E, Munn Z, editors. JBI Manual for Evidence Synthesis. JBI; 2020 [cited 2021 Mayo 16]. Available from: https://wiki.jbi.global/display/MANUAL/ Chapter+5\%3A+Systematic+reviews+of+prevalence+and+incidence

16. Moher D, Liberati A, Tetzlaff J, Altman DG. Preferred reporting items for systematic reviews and meta-analyses: the PRISMA statement. PLoS Med. 2009;6(7):e1000097.

17. Munn Z, Moola S, Lisy K, Riitano D, Tufanaru C. Methodological guidance for systematic reviews of observational epidemiological studies reporting prevalence and cumulative incidence data. Int J Evid Based Healthc. 2015;13(3):147-53.

18. Tufanaru C, Munn Z, Stephenson M, Aromataris E. Fixed or random effects meta-analysis? Common methodological issues in systematic reviews of effectiveness. Int J Evid Based Healthc. 2015;13(3):196207.

19. Higgins JP, Thomas J, Chandler J, Cumpston M, Li T, Page MJ, et al. Cochrane Handbook for Systematic Reviews of Interventions version 6.0 (Updated July 2019). United Kingdom: Cochrane; 2019 [cited 2021 May 16]. Available from: https://training.cochrane.org/handbook

20. Bar-Zeev N, Inglesby T. COVID-19 vaccines: early success and remaining challenges. Lancet. 2020;396(10255):868-9. 